\title{
Diacronie
}

Studi di Storia Contemporanea

$N^{\circ} 12,4 \mid 2012$

Sulle tracce delle idee

\section{Simone Neri Serneri (a cura di), Verso la lotta armata. La politica della violenza nella sinistra radicale degli anni Settanta}

\section{Giorgio Sacchetti}

\section{(2) OpenEdition \\ Journals}

Edizione digitale

URL: http://journals.openedition.org/diacronie/2442

DOI: $10.4000 /$ diacronie.2442

ISSN: 2038-0925

Editore

Association culturelle Diacronie

Notizia bibliografica digitale

Giorgio Sacchetti, «Simone Neri Serneri (a cura di), Verso la lotta armata. La politica della violenza nella sinistra radicale degli anni Settanta », Diacronie [Online], № 12, 4 | 2012, documento 17, Messo online il 29 décembre 2012, consultato il 24 septembre 2020. URL : http://journals.openedition.org/diacronie/ 2442 ; DOI : https://doi.org/10.4000/diacronie.2442 


\title{
RECENSIONE:
}

\section{Simone NERI SERNERI (a cura di), Verso la} lotta armata. La politica della violenza nella sinistra radicale degli anni Settanta, Bologna, Il Mulino, 2012, 404 pp.

\begin{abstract}
Una riflessione storiografica sull'esercizio della violenza dovrà spingersi oltre le opposte, ma riduttive, tendenze ad addebitarla all'esaltazione ideologica o alla degenerazione meramente criminale $o$, invece, a considerarla esito pur deprecabile delle contingenze politiche $[\ldots]^{1}$
\end{abstract}

S. Neri Serneri

Il passaggio all'attività storiografica propriamente detta per quel che riguarda gli accadimenti dell'era contemporanea, è in genere preceduto da una naturale fase intermedia di narrazioni memorialistiche e mediatiche, quasi sempre prodotte o da protagonisti o comunque da parti in causa interessate. Queste letture particolari sono destinate poi a diventare esse stesse fonti preziose per gli storici. Succede sempre così. Sulla connessione tra violenza politica e prassi radicali nella sinistra degli anni Settanta, tema di ricerca approfondito in questo bel volume curato da Neri Serneri, si è dovuto invece registrare - ancora nell'ultimo decennio - una fatica maggiore degli studiosi (salvo qualche rarissima eccezione) ad entrare con i ferri del mestiere in medias res. Quest'opera, che raccoglie una parte consistente delle relazioni presentate al convegno Violenza politica e lotta armata nella sinistra italiana degli anni Settanta (Firenze, 27-28 maggio 2010), promosso dall'Istituto Storico della Resistenza in Toscana, fornisce - «superando i recinti delle memorie e le genealogie manichee» ${ }^{2}-$

\footnotetext{
${ }^{1}$ NERI SERNERI, Simone (a cura di), Verso la lotta armata. La politica della violenza nella sinistra radicale degli anni Settanta, Bologna, Il Mulino, 2012, p. 24

${ }^{2}$ Dalla quarta di copertina del volume.
} 
una prima plausibile, per quanto interlocutoria, risposta ai tanti interrogativi sulla genesi fenomenologica della lotta armata in quegli anni. Perché una mobilitazione generazionale, mai vista prima in quelle dimensioni, alimentata da profonde tensioni ideali e da istanze di sacrosanta giustizia sociale, incrocia la violenza come metodo e strumento dell'agire politico? Quale sfera di riferimento ambientale si deve individuare per spiegare il corto circuito avvenuto tra le progettualità dei vari soggetti e le culture antropologiche di riferimento? Come può, infine, essere maturata la scelta collettiva/individuale di militarizzare il conflitto?

Trentasei sono i gruppi censiti nello studio. Il fenomeno ha investito prevalentemente il nord-ovest del paese, in particolare le aree metropolitane industriali di Torino, Milano, Genova; poi è stata la volta di Roma (cuore dello Stato), quindi la Toscana, il Veneto, l'Emilia e la Campania. Ventimila inquisiti, migliaia di incarcerati, 1.119 sono state le vittime della violenza politica nel periodo 1969-1982, di cui 351 morti. Esito tragico di azioni cosiddette dimostrative, attentati e sabotaggi, violenze, ferimenti, scontri a fuoco, gambizzazioni varie, sequestri di persona... Il volume, che ha tra gli altri il pregio di aggiornare e tracciare ex-novo una mappa italiana del fenomeno (contesti sociali, politici, territori,...), si ripartisce in tre sezioni che potrebbero costituire benissimo altrettanti filoni d'indagine, traccia utile per il prosieguo del dibattito e di futuri ulteriori auspicabili contributi scientifici. Gli autori hanno tutti approfondito in passato i temi: Discutere il caso italiano 3 (con contributi di Simone Neri Serneri, Monica Galfré, Marco Grispigni, Marco Scavino); Retoriche e legittimazione della violenza 4 (Silvia Casilio, Barbara Armani, Isabelle Sommier); Luoghi, pratiche, contesti5 (Christian G. De Vito, Guido Panvini, Lorenzo Bosi e Donatella della Porta, Vincenzo Filetti, David Serafino).

Pur considerando, nel complesso, periodizzante la cesura di metà anni Settanta (che coincide con la fine del cosiddetto "ottimismo rivoluzionario" e degli "anni d'oro" evocati da Hobsbawm $^{6}$ ), queste pagine valutano nell'insieme come suscettibili di un'unica analisi il lungo Sessantotto italiano (un po' marxista e un po' libertario) ed il Settantasette (libertario e "situazionista"), con risultati - ci pare - interessanti . Il 1974 fu dunque "anno di passaggio" ed il successivo biennio "punto di non ritorno". Nel contempo cadde il dogma della centralità operaia mentre comparirono sullo scenario politico e sociale nuovi soggetti protagonisti. Certo, le cesure temporali su questa specifica tematica non rappresentano ancora un chiaro punto di riferimento del lavoro

3 NERI SERNERI (a cura di), op. cit., pp. 11-205.

${ }^{4}$ Ibidem, pp. 207-283.

5 Ibidem, pp. 285-389.

${ }^{6}$ HOBSBAWM, Eric J., Il secolo breve 1914-1991, Milano, Rizzoli, 2006, pp. 303-336. 
storiografico. Esse risentono di letture avulse e generaliste sul decennio, fatte spesso attraverso l'uso di categorie banalizzanti - da talk show, si potrebbe dire - quali "opposti estremismi”, "anni di piombo" e così via. Dall'altra parte si riscontra in questo volume uno sforzo apprezzabile per superare certe letture semplicistiche coeve, tendenti ad esaltare la presunta natura difensiva delle violenze messe in atto dalla sinistra radicale, qualificate come mera risposta al neosquadrismo fascista oppure alle connivenze dello Stato nelle stragi. Ma piazza Fontana rimane, con ogni evidenza, il punto focale di tutta la storia. Mentre fra le tracce concrete, profonde e durature, si annoverano: il rapporto consolidato fra lotta armata e detenuti politicizzati; la nuova collocazione di un ceto politico "fabbrichista" proveniente da precedenti percorsi in Potere Operaio e Lotta Continua. Antifascismo militante e ricorso alla mitologia resistenziale rimangono comunque chiavi di lettura che ci inducono ad inquadrare sempre la violenza politicamente motivata in un'analisi di lungo periodo. I percorsi individuali di mobilitazione si dipanano su tre dimensioni: l’ideologia, la valutazione politica strumentale, la spinta solidale emotiva. Le uccisioni di Aldo Moro, di Guido Rossa e del giudice Alessandrini innescano il processo di dissoluzione di un percorso militarista e violento caratterizzato dall'isolamento e dalla distanza siderale da quelle istanze di matrice libertaria che avevano dato l'incipit ai movimenti del Sessantotto e del Settantasette.

Nella genesi del nesso tra pratiche violente - intese in senso lato - e lotta armata è da valutare con la massima attenzione la coesistenza di due anime nei movimenti, una vera costante nel decennio, ossia l'antinomia autoritario/antiautoritario. In particolare ci sembra di non scarso rilievo, nell'ultimo scorcio dei Settanta, la divaricazione di contenuti tra "lottarmatismo" e pratica della controcultura radicale, tra violenza autoreferenziata e happening di sovversione dei linguaggi. E su questi ultimi aspetti, che certo riguardano tutto il decennio, il volume non manca di indagare a dovere nel "perimetro dell'auto-rappresentazione" quella "retorica della violenza" che ha pervaso tutti i movimenti, stando almeno all'analisi delle fonti a stampa. Talvolta la rappresentazione del conflitto, con le sue inevitabili "gogne informative", ha avuto un'importanza maggiore del conflitto stesso. "Parole armate" che hanno avuto comunque peso. Sulla disfatta, non solo militare, dell'opzione armata di quegli anni ci sentiamo di condividere le conclusioni del curatore:

Proprio quando riuscirono a esprimere la loro massima potenzialità offensiva, le organizzazioni armate non seppero accompagnarla con una corrispondente qualificazione degli obiettivi politico-militari [...] e subirono la crescente capacità 
repressiva delle autorità di pubblica sicurezza e della magistratura. In sostanza, si dimostrarono definitivamente incapaci di spostare a proprio favore i rapporti di forza sul terreno politico, ammesso che mai ne avessero avuto possibilità $[\ldots .$.$] .$

Rimane, insieme alla tragedia immane delle vittime, «il fallimento di una scelta politica, a lungo coltivata, il cui peso sulle vicende italiane di quel cruciale decennio merita di essere scandagliato più a fondo» 8 .

Ci sarebbe infine una marginale osservazione da fare, forse dettata dalla visuale tutta "specialistica" del recensore. Nell'opera non è offerta la giusta evidenza alla componente anarchica. E con questo non ci si vuole riferire soltanto alla piccola formazione armata "Azione Rivoluzionaria", quanto piuttosto al coinvolgente dibattito sulla violenza in ambito libertario oggi desumibile dalle fonti soggettive (Archivio Storico della Federazione Anarchica Italiana in primis). Si tratta di filoni ideali che in quegli anni ebbero un peso nella rivolta giovanile italiana e nel suo accidentato prosieguo, quanto meno in termini di esprit per dirla con Jean Maitron?.

7 NERI SERNERI, Simone (a cura di), Verso la lotta armata. La politica della violenza nella sinistra radicale degli anni Settanta, Bologna, Il Mulino, 2012, pp. 60-61

8 Ibidem.

9 MAITRON, Jean, La pensée anarchiste traditionnelle et la révolte des jeunes, in Anarchici e Anarchia nel mondo contemporaneo. Atti del Convegno promosso dalla Fondazione Luigi Einaudi (Torino, 5, 6 e 7 dicembre 1969), Torino, Fondazione Luigi Einaudi, 1971, pp. 543-578. 


\section{* L'autore}

Giorgio Sacchetti è dottore di ricerca in Storia del movimento sindacale, cultore della materia presso la cattedra di Storia dei partititi e dei movimenti politici all'Università di Trieste. Già direttore della «Rivista Storica dell'Anarchismo»; membro dei comitati scientifici Archivio fam. Berneri - A. Chessa (Reggio Emilia) e «Germinal - Revista de Estudios Libertarios» (Madrid); redattore del Dizionario Biografico degli anarchici italiani (BFS, 2003-2004). Si occupa di labour history e di culture libertarie del Novecento. L'ultimo volume da lui pubblicato è Sovversivi e squadristi. 1921: alle origini della guerra civile in provincia di Arezzo, Roma, Aracne, 2010.

URL: < http://www.studistorici.com/progett/autori/\#Sacchetti >

\section{Per citare questo articolo:}

SACCHETTI, Giorgio, «Recensione: Simone NERI SERNERI (a cura di), Verso la lotta armata. La politica della violenza nella sinistra radicale degli anni Settanta, Bologna, II Mulino, 2012, 404 pp.», Diacronie. Studi di Storia Contemporanea: Sulle tracce delle idee, 29/12/2012,

URL:< http://www.studistorici.com/2012/12/29/sacchetti_numero_12/ >

Diacronie Studi di Storia Contemporanea $\beta$ www.diacronie.it

Risorsa digitale indipendente a carattere storiografico. Uscita trimestrale. redazione.diacronie@hotmail.it

Comitato di redazione: Marco Abram - Jacopo Bassi - Luca Bufarale - Gianluca Canè - Alessandro Cattunar - Alice De Rensis - Barbara Galimberti - Deborah Paci - Fausto Pietrancosta - Matteo Tomasoni - Luca Zuccolo

Diritti: gli articoli di Diacronie. Studi di Storia Contemporanea sono pubblicati sotto licenza Creative Commons 2.5. Possono essere riprodotti a patto di non modificarne i contenuti e di non usarli per fini commerciali. La citazione di estratti è comunque sempre autorizzata, nei limiti previsti dalla legge. 